\title{
Valley splitting of single-electron Si MOS quantum dots
}

John King Gamble, Patrick Harvey-Collard, N. Tobias Jacobson, Andrew D. Baczewski, Erik Nielsen, Leon Maurer, Inès Montaño, Martin Rudolph, M. S. Carroll, C. H. Yang, A. Rossi, A. S. Dzurak, and Richard P. Muller

Citation: Appl. Phys. Lett. 109, 253101 (2016); doi: 10.1063/1.4972514

View online: http://dx.doi.org/10.1063/1.4972514

View Table of Contents: http://aip.scitation.org/toc/apl/109/25

Published by the American Institute of Physics

\section{Articles you may be interested in}

Ultra-fine metal gate operated graphene optical intensity modulator

Appl. Phys. Lett. 109, 251101251101 (2016); 10.1063/1.4972306

Investigation of electron irradiation-induced magnetism in layered MoS2 single crystals

Appl. Phys. Lett. 109, 252403252403 (2016); 10.1063/1.4971192

Low p-type contact resistance by field-emission tunneling in highly Mg-doped GaN

Appl. Phys. Lett. 109, 252101252101 (2016); 10.1063/1.4972408

Route toward high-speed nano-magnonics provided by pure spin currents

Appl. Phys. Lett. 109, 252401252401 (2016); 10.1063/1.4972244

Toward the III-V/Si co-integration by controlling the biatomic steps on hydrogenated $\mathrm{Si}(001)$

Appl. Phys. Lett. 109, 253103253103 (2016); 10.1063/1.4972394

Nanoscale deformations in graphene by laser annealing

Appl. Phys. Lett. 109, 253102253102 (2016); 10.1063/1.4972845

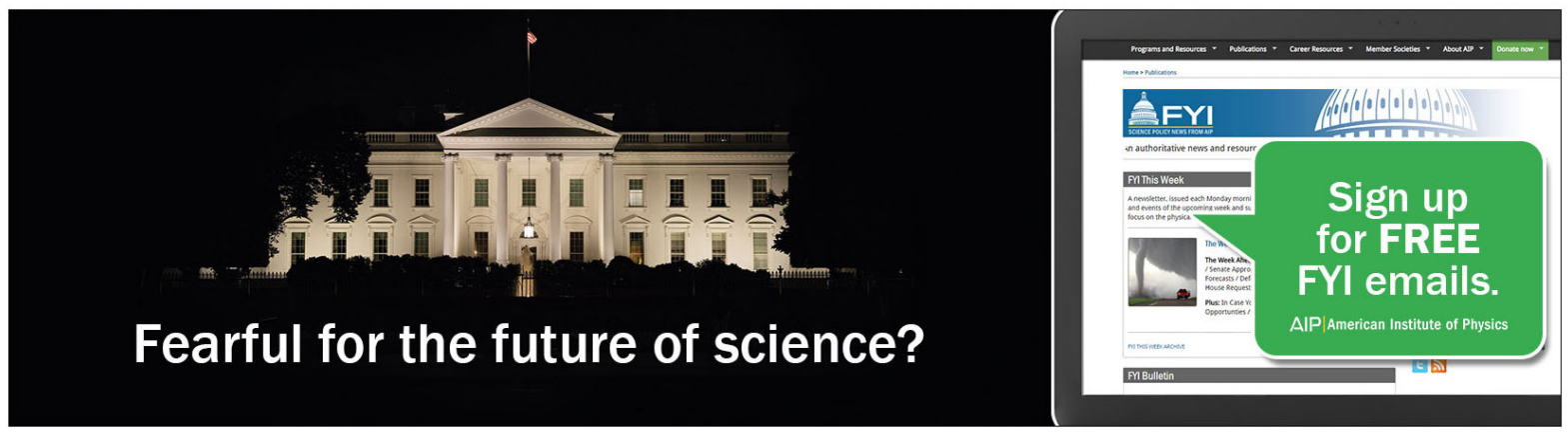




\title{
Valley splitting of single-electron Si MOS quantum dots
}

\author{
John King Gamble, ${ }^{1, a)}$ Patrick Harvey-Collard, ${ }^{2,3,4}$ N. Tobias Jacobson, ${ }^{1}$ \\ Andrew D. Baczewski, ${ }^{1}$ Erik Nielsen, ${ }^{4}$ Leon Maurer, ${ }^{1}$ Inès Montaño, ${ }^{4}$ Martin Rudolph, ${ }^{4}$ \\ M. S. Carroll, ${ }^{4}$ C. H. Yang, ${ }^{5}$ A. Rossi, ${ }^{6}$ A. S. Dzurak, ${ }^{5}$ and Richard P. Muller ${ }^{1}$ \\ ${ }^{1}$ Center for Computing Research, Sandia National Laboratories, Albuquerque, New Mexico 87185, USA \\ ${ }^{2}$ Département de physique, Université de Sherbrooke, Sherbrooke, Quebec JIK 2RI, Canada \\ ${ }^{3}$ Institut quantique, Université de Sherbrooke, Sherbrooke, Quebec JIK 2R1, Canada \\ ${ }_{5}^{4}$ Sandia National Laboratories, Albuquerque, New Mexico 87185, USA \\ ${ }^{5}$ Australian Research Council Centre of Excellence for Quantum Computation and Communication \\ Technology, School of Electrical Engineering and Telecommunications, The University of New South Wales, \\ Sydney 2052, Australia \\ ${ }^{6}$ Cavendish Laboratory, University of Cambridge, Cambridge CB3 OHE, United Kingdom
}

(Received 11 October 2016; accepted 1 December 2016; published online 19 December 2016)

\begin{abstract}
Silicon-based metal-oxide-semiconductor quantum dots are prominent candidates for high-fidelity, manufacturable qubits. Due to silicon's band structure, additional low-energy states persist in these devices, presenting both challenges and opportunities. Although the physics governing these valley states has been the subject of intense study, quantitative agreement between experiment and theory remains elusive. Here, we present data from an experiment probing the valley states of quantum dot devices and develop a theory that is in quantitative agreement with both this and a recently reported experiment. Through sampling millions of realistic cases of interface roughness, our method provides evidence that the valley physics between the two samples is essentially the same. Published by AIP Publishing. [http://dx.doi.org/10.1063/1.4972514]
\end{abstract}

Qubits based on isolated electron spins in semiconductors are one of the earliest proposals for a quantum information processing architecture, ${ }^{1}$ where electrons are confined to zero-dimensional quantum dots (QDs) via electrostatic gates patterned on the surface of a semiconductor heterostructure. ${ }^{2}$ These isolated electrons resemble artificial atoms, and are very versatile, supporting several qubit encoding schemes. ${ }^{3-5}$ $\mathrm{Si}^{6-9}$ is a promising candidate for these qubits due to excellent electronic spin coherence times that can easily range to seconds. ${ }^{10}$

A complication in $\mathrm{Si}$ arises from its band structure; in the bulk, the conduction band has six degenerate minima, called valleys. This valley degeneracy is broken by the sharp material interfaces present in heterostructures, resulting in a low-lying manifold of additional electronic states. The presence of these states can be either a benefit ${ }^{11,12}$ or a drawback, ${ }^{13,14}$ but understanding and being able to predictably engineer the valley physics in quantum dots is important for developing qubits.

For these reasons, the valley physics of silicon has been the subject of intense study over the past decade. Researchers have used effective mass theory, ${ }^{15}$ atomistic pseudopotentials, ${ }^{16}$ and atomistic tight binding ${ }^{17-20}$ to make predictions of the energy gap between the lowest two valley states, termed the valley splitting, in a variety of experimentally relevant scenarios. These studies indicate that disorder in the heterostructure interface dramatically influences the valley splitting, ${ }^{21,22}$ leading to the unfortunate conclusion that valley splitting may vary substantially amongst nominally identical devices.

Recently, experiments have advanced to the point where it is possible to track valley splitting as a function of applied

a)jkgambl@sandia.gov electrostatic biases while maintaining single-electron dot occupation. ${ }^{23}$ In this work, we present a second measurement on a device with a significantly different design and fabrication process. We then develop a non-perturbative, multivalley effective mass theory that can directly simulate both experiments. We find similar, predictable behavior in the tuning of the valley splitting. Our theory enables efficient high-throughput numerical sampling of random interfaces, achieving quantitative agreement with experiment and providing a substantial improvement upon previous work.

The experiments were performed on two different metal-oxide-semiconductor (MOS) quantum dot (QD) samples. Electrodes patterned on the top of the device were used to provide confinement, isolating a single electron in a QD. The first device, depicted in Fig. 1(a), is a single-layer gated wire geometry fabricated at Sandia National Laboratories (SNL). ${ }^{24}$ The second device, shown in Fig. 1(b), is a threelayer design fabricated at the University of New South Wales (UNSW). ${ }^{23}$

The valley splitting of a single-electron quantum dot was measured. In tightly confined quantum dots like those considered here, the first excited state carries a valley-like degree of freedom, ${ }^{25}$ so the valley splitting is given by the difference between the first excited and ground state of the quantum dot: $E_{V S}=E_{1}-E_{0}$. Here, confinement to a narrow sheet next to the interface splits the six-fold degenerate conduction band minima of bulk silicon into a low-lying doublet and an excited quadruplet. The doublet, whose conduction band minima lie along the $\pm \hat{z}$ directions in momentum space, is further split by the sharp oxide interface potential. By changing the voltages on the control electrodes while compensating to ensure the quantum dot remains in the single-electron regime, the electronic wavefunction can be forced to penetrate more into 


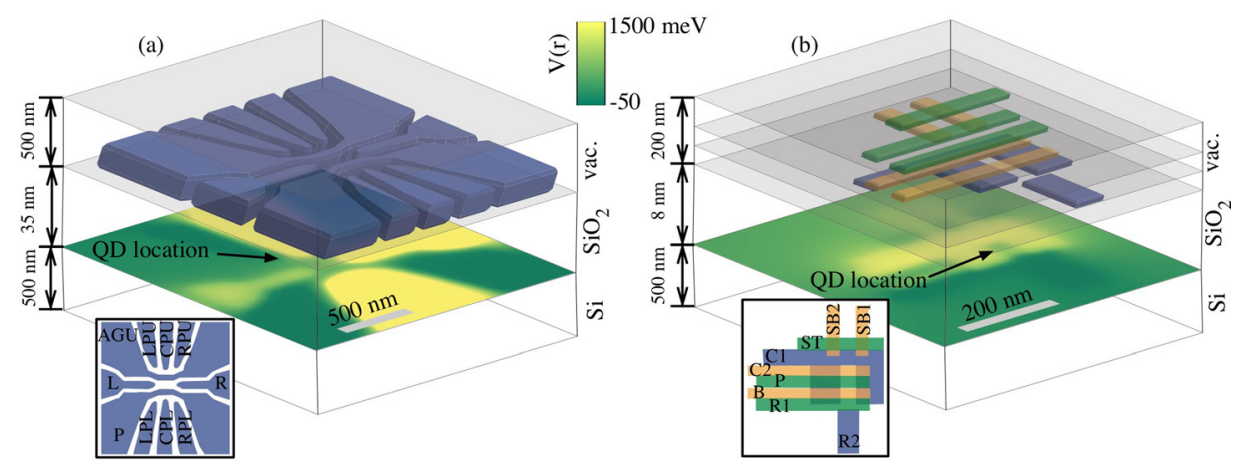

FIG. 1. Schematics of both devices used in this work. (a) The device fabricated at SNL, ${ }^{24}$ comprising a single layer of $200 \mathrm{~nm} \mathrm{n}$-doped poly-Si electrodes on $35 \mathrm{~nm}$ oxide. The single quantum dot studied here is formed at the Si-oxide interface as indicated. (b) The device fabricated at the UNSW, ${ }^{23}$ comprising three layers of aluminum electrodes on $8 \mathrm{~nm}$ oxide. The gates are separated by a thin layer of thermally grown $\mathrm{Al}_{\mathrm{x}} \mathrm{O}_{\mathrm{y}}$. The potential energy surface $V(r)$ witnessed by electrons $3 \mathrm{~nm}$ beneath the oxide is shown, the result of self-consistent Thomas-Fermi calculations (see main text for additional details).

the oxide barrier, effectively tuning the valley splitting as a function of voltage configuration.

In the SNL experiment, the valley splitting is measured using a pulsed gate spectroscopy technique. ${ }^{4,26}$ The quantum dot is tuned to the single electron regime, and the tunnel rate to the lead is adjusted to roughly $10 \mathrm{kHz}$. Then, using a square pulse on the CPL gate (Fig. 1) of varying frequency and mean voltage, the excited one-electron states of the quantum dot are probed by monitoring the average quantum dot occupancy with the charge sensor. ${ }^{21}$ For a small range of frequencies, both the ground state and the first excited state can be seen, even if they have similar tunnel rates, allowing the measurement of their energy separation. Gate voltage differences are converted to energy using a gate lever-arm, which is calibrated through a temperature dependence measurement. The procedure is repeated for multiple gate voltage configurations. In particular, the $\mathrm{P}$ gate has the most influence on the electrical field perpendicular to the oxide interface, and thus has a large influence on the valley splitting as well. The gate voltages were thus chosen to explore a large range of $\mathrm{P}$ voltages.

In the UNSW experiment, reported in Ref. 23 , two techniques were used to measure the valley splitting. For small plunger gate voltages $V_{p}$, a spin-relaxation hotspot, for which the electron $T_{1}$ time is minimized when the valley splitting is commensurate with the Zeeman splitting of the device, was used. For larger values of $V_{p}$, pulsed-gate magnetospectroscopy was used. For details regarding the measurement technique, see Ref. 23.

Since the valley splitting depends sensitively on the electrostatics of the problem, here, we take a multi-stage approach to our calculations. First, we perform self-consistent Thomas-Fermi simulations ${ }^{27}$ of the devices under the experimental voltage configurations. In the leads of the device, this simulation captures the effect of dynamic screening, using COMSOL Multiphysics with a 2D density of states to self-consistently model charge accumulation at the oxidesilicon interface. Nearby the quantum dots, we exclude selfconsistent accumulation, since the devices are experimentally tuned to the single-electron regime. We note that when tuned using the experimental voltages, the UNSW device simulation exhibited a clear quantum dot potential, so the dot accumulation region was readily excluded from accumulation. The
SNL simulation did not show a clear dot-lead separation, so the choice of the exclusion zone (which then generates a dot confinement potential) was somewhat ambiguous. A possible explanation for this discrepancy is non-uniform fixed charge in the real device but not in the simulation. The output of the electrostatic simulations is then fed into a non-perturbative multi-valley effective mass theory. ${ }^{28}$ For implementation details, see the supplementary material.

We simulated both experiments using multi-valley effective mass theory, as described above. First, we restricted ourselves to the case of a flat interface. Our electrostatic simulations do not take the threshold voltage shift due to oxide charge into account directly. To mimic various thresholds, we apply a uniform voltage shift $V_{0}$ to all the electrodes in the simulation relative to the Fermi level, so that the voltages in the simulation $V_{\text {sim }}=V_{\text {exp }}+V_{0}$, where $V_{\text {exp }}$ are the experimental voltages. This causes a shift in when the theory predicts carrier accumulation (e.g., $V_{0}=-1.0 \mathrm{~V}$ implies carrier accumulation beginning at applied biases of $+1.0 \mathrm{~V}$ ).

In Fig. 2, we show the results of these calculations. For both devices, the computed slope of valley splitting vs. voltage agrees well with the experiment. For no voltage offset $\left(V_{0}=0\right)$, there is a pronounced uniform shift of $0.1-0.2 \mathrm{meV}$,
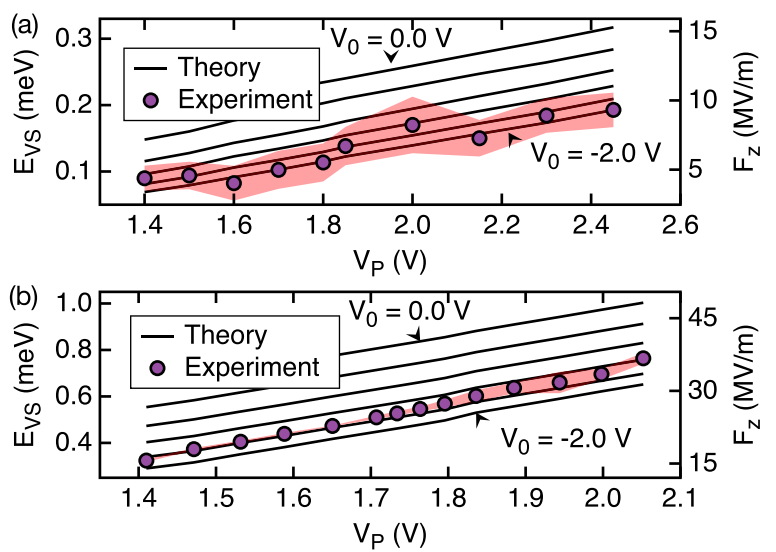

FIG. 2. Measured and computed valley splittings. (a) Results for the SNL device. $^{24}$ The experimental data (dots) are shown with measurement error bars as a band. (b) Results from the UNSW device. ${ }^{23}$ The experimental data and error bars were reported in Ref. 23; the theory results are new here. In both cases, the best-fit voltage offset is about $V_{0}=-1.8 \mathrm{~V}$. The effective vertical electric fields plotted on the right axis are computed using a vertical field vs. valley splitting relationship (see supplementary material). 
with the theory overestimating the valley splitting. This is not unexpected: an offset was previously observed, ${ }^{23}$ where it was attributed to the interface disorder. In that previous work, the offset was reported to be considerably larger than what we find here $(1 \mathrm{meV})$. Our model directly computes the valley splitting from the full electrostatic potential, including the important fringing, non-uniform vertical field. In contrast, Ref. 23 extracted an approximate vertical electric field from technology computer aided design (TCAD) calculations and fed the results into previous simple model system calculations of valley splitting. To make more direct contact with previous results, we can translate our valley splitting results into an effective vertical electric field (i.e., the vertical electric field that, in an ideal model system, would explain the valley splitting). We do this using the valley splitting vs. vertical electric field results shown in Fig. 1(b) of the supplementary material, and we show the effective vertical electric fields in Fig. 2 on the right axis. The UNSW device exhibits higher valley splittings than the SNL device mainly due to thinner oxide thickness, smaller device features, and larger applied voltages, all of which serve to raise the effective vertical electric field.

Despite obtaining excellent experimental agreement at $V_{0}$ $=-1.8 \mathrm{~V}$, thresholds in these devices are typically between 0.1 and $1.0 \mathrm{~V}$. Hence, from experiment, we expect to need to include a compensating offset of $V_{0}=-0.1$ to $-1.0 \mathrm{~V}$ in our simulations. To investigate this apparent discrepancy, in Fig. 3, we show the effect of disordered interfaces on the valley splitting. We parameterize the interface using a Gaussian correlation function and a two-parameter correlation length and root-mean-squared (RMS) roughness model. ${ }^{22}$ We sample these parameters over a $20 \times 20$ grid, with 65 random realizations per point. For each case, we choose a voltage offset $V_{0}$ and then compute the valley splitting for the experimental voltages. We report the worst-case relative error $\epsilon_{\mathrm{wc}}$ $=\max _{V_{p}}\left(\left|E_{V S}^{\exp }\left(V_{p}\right)-E_{V S}^{\text {theory }}\left(V_{p}\right)\right| /\left|E_{V S}^{\exp }\left(V_{p}\right)\right|\right)$ with respect

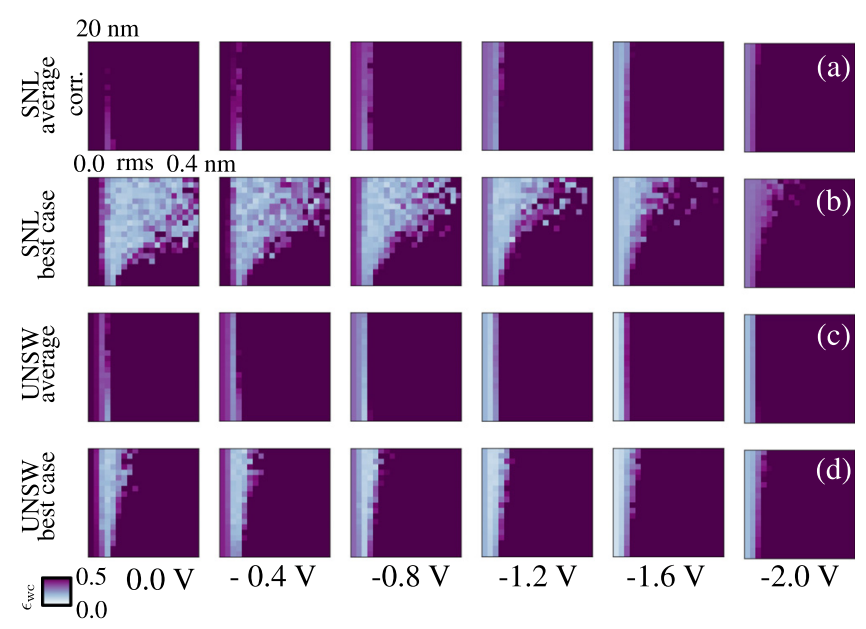

FIG. 3. Worst case relative error $\epsilon_{\mathrm{wc}}$ of valley splitting with respect to the experiment for disordered interfaces. Each plot shows a 2D sweep over correlation length and root-mean-squared (RMS) roughness. Offset voltages between $V_{0}=0.0 \mathrm{~V}$ and $V_{0}=-2.0 \mathrm{~V}$ are shown as columns, which correspond to the lines shown in Fig. 2. Since we do not know the actual experimental interface configurations, we report $\epsilon_{\mathrm{wc}}$ averaged over the interface realizations in panels (a) and (c) and for the best interfaces in panels (b) and (d). These results show that by increasing the magnitude of disorder, lower threshold voltages produce valley splittings compatible with experiment. to the experimental valley splittings, where $E_{V S}^{\exp }\left(V_{p}\right)$ and $E_{V S}^{\text {theory }}\left(V_{p}\right)$ are the measured and predicted valley splittings at voltage $V_{p}$, respectively.

For both the SNL and UNSW devices, we show $\epsilon_{\mathrm{wc}}$ averaged over the 65 interface realizations as well as the result for the best interface. In both cases, we found disordered interfaces that are consistent with the lower threshold voltages observed in experiment as well as realistic MOS interface parameters of RMS roughness $\sim 0.1 \mathrm{~nm}$ (Ref. 29) and a wide range of correlation lengths. This shows that the introduction of realistic disorder is sufficient to solve the apparent discrepancy between theoretical and experimental threshold voltages noted in Fig. 2.

In this work, we analyzed the valley splitting for two distinct MOS devices: a single-layer gated-wire design fabricated at SNL and a multi-layer device fabricated at UNSW. Despite superficially appearing to have very different valley splitting properties, detailed multi-valley effective mass theory calculations of the valley splitting, directly incorporating the potential energy landscape, revealed that geometric differences are likely responsible for the differences and that the valley physics is consistent across the two devices. By introducing a voltage offset of $-1.8 \mathrm{~V}$ to mimic threshold voltage, we obtained quantitative agreement with experiment. Since this value is larger than what is typically seen in experiment, we implemented a non-perturbative disordered interface model to attempt to explain this discrepancy. Through this, we found plausible interface roughness parameters that lead to realistic threshold voltages.

Overall, our results suggest that MOS quantum dots are a promising qubit platform. Since excessively small valley splitting is problematic for qubit operation, being able to reliably tune and design for large valley splitting is critical for successful qubit operation. Here, we have put forward evidence that MOS single-electron valley splitting is both tunable and predictable, opening the door to further design and optimization of robust qubits.

See supplementary material for details on multi-valley effective mass theory, convergence and Bloch function information, more details on the electrostatic finite element simulations, and details on the computation of the disorder matrix elements.

The authors acknowledge useful discussions with F. Mohiyaddin and M. Usman. Sandia National Laboratories is a multi-program laboratory managed and operated by Sandia Corporation, a wholly owned subsidiary of Lockheed Martin Corporation, for the U.S. Department of Energy's National Nuclear Security Administration under Contract No. DEAC04-94AL85000. J.K.G. gratefully acknowledges support from the Sandia National Laboratories Truman Fellowship Program, which is funded by the Laboratory Directed Research and Development (LDRD) program. This work was performed, in part, at the Center for Integrated Nanotechnologies, an Office of Science User Facility operated for the U.S. Department of Energy (DOE) Office of Science. C.H.Y. and A.S.D. acknowledge support from the Australian Research Council (CE11E0001017), the U.S. Army Research Office (W911NF-13-1-0024) and the NSW 
Node of the Australian National Fabrication Facility. A.R. acknowledges support from the European Union's Horizon 2020 research and innovation programme under the Marie Skłodowska-Curie Grant Agreement No. 654712 (SINHOPSI).

${ }^{1}$ D. Loss and D. P. DiVincenzo, Phys. Rev. A 57, 120 (1998).

${ }^{2}$ R. Hanson, J. R. Petta, S. Tarucha, and L. M. K. Vandersypen, Rev. Mod. Phys. 79, 1217 (2007).

${ }^{3}$ D. P. DiVincenzo, D. Bacon, J. Kempe, G. Burkard, and K. B. Whaley, Nature 408, 339 (2000).

${ }^{4}$ J. R. Petta, A. C. Johnson, J. M. Taylor, E. A. Laird, A. Yacoby, M. D. Lukin, C. M. Marcus, M. P. Hanson, and A. C. Gossard, Science 309, 2180 (2005).

${ }^{5}$ Z. Shi, C. B. Simmons, J. R. Prance, J. K. Gamble, T. S. Koh, Y.-P. Shim, X. Hu, D. E. Savage, M. G. Lagally, M. A. Eriksson, M. Friesen, and S. N. Coppersmith, Phys. Rev. Lett. 108, 140503 (2012).

${ }^{6}$ B. M. Maune, M. G. Borselli, B. Huang, T. D. Ladd, P. W. Deelman, K. S. Holabird, A. A. Kiselev, I. Alvarado-Rodriguez, R. S. Ross, A. E. Schmitz, M. Sokolich, C. A. Watson, M. F. Gyure, and A. T. Hunter, Nature 481, 344 (2012).

${ }^{7}$ J. J. Pla, K. Y. Tan, J. P. Dehollain, W. H. Lim, J. J. L. Morton, D. N. Jamieson, A. S. Dzurak, and A. Morello, Nature 489, 541 (2012).

${ }^{8}$ D. Kim, Z. Shi, C. Simmons, D. Ward, J. Prance, T. S. Koh, J. K. Gamble, D. Savage, M. Lagally, M. Friesen et al., Nature 511, 70 (2014).

${ }^{9}$ M. Veldhorst, C. H. Yang, J. C. C. Hwang, W. Huang, J. P. Dehollain, J. T. Muhonen, S. Simmons, A. Laucht, F. E. Hudson, K. M. Itoh, A. Morello, and A. S. Dzurak, Nature 526, 410 (2015).

${ }^{10}$ F. A. Zwanenburg, A. S. Dzurak, A. Morello, M. Y. Simmons, L. C. L. Hollenberg, G. Klimeck, S. Rogge, S. N. Coppersmith, and M. A. Eriksson, Rev. Mod. Phys. 85, 961 (2013).

${ }^{11}$ V. N. Smelyanskiy, A. G. Petukhov, and V. V. Osipov, Phys. Rev. B 72, 081304 (2005).

${ }^{12}$ D. Culcer, A. L. Saraiva, B. Koiller, X. Hu, and S. Das Sarma, Phys. Rev. Lett. 108, 126804 (2012).
${ }^{13}$ S. Goswami, K. A. Slinker, M. Friesen, L. M. McGuire, J. L. Truitt, C. Tahan, L. J. Klein, J. O. Chu, P. M. Mooney, D. W. van der Weide, R. Joynt, S. N. Coppersmith, and M. A. Eriksson, Nat. Phys. 3, 41 (2007).

${ }^{14}$ D. Culcer, L. Cywiński, Q. Li, X. Hu, and S. Das Sarma, Phys. Rev. B 80, 205302 (2009).

${ }^{15}$ M. Friesen, S. Chutia, C. Tahan, and S. Coppersmith, Phys. Rev. B 75, 115318 (2007).

${ }^{16}$ L. Zhang, J.-W. Luo, A. Saraiva, B. Koiller, and A. Zunger, Nat. Commun. 4, 2396 (2013).

${ }^{17}$ T. Boykin, G. Klimeck, M. Friesen, S. Coppersmith, P. von Allmen, F. Oyafuso, and S. Lee, Phys. Rev. B 70, 165325 (2004).

${ }^{18}$ N. Kharche, M. Prada, T. B. Boykin, and G. Klimeck, Appl. Phys. Lett. 90, 092109 (2007).

${ }^{19}$ R. Rahman, J. Verduijn, N. Kharche, G. P. Lansbergen, G. Klimeck, L. C. L. Hollenberg, and S. Rogge, Phys. Rev. B 83, 195323 (2011).

${ }^{20}$ Z. Jiang, N. Kharche, T. Boykin, and G. Klimeck, Appl. Phys. Lett. 100, 103502 (2012).

${ }^{21}$ Z. Shi, C. B. Simmons, J. R. Prance, J. King Gamble, M. Friesen, D. E. Savage, M. G. Lagally, S. N. Coppersmith, and M. A. Eriksson, Appl. Phys. Lett. 99, 233108 (2011).

${ }^{22}$ D. Culcer, X. Hu, and S. Das Sarma, Phys. Rev. B 82, 205315 (2010).

${ }^{23}$ C. H. Yang, A. Rossi, R. Ruskov, N. S. Lai, F. A. Mohiyaddin, S. Lee, C. Tahan, G. Klimeck, A. Morello, and A. S. Dzurak, Nat. Commun. 4, 2069 (2013).

${ }^{24}$ P. Harvey-Collard, N. T. Jacobson, M. Rudolph, J. Dominguez, G. A. T. Eyck, J. R. Wendt, T. Pluym, J. K. Gamble, M. P. Lilly, M. Pioro-Ladrière et al., preprint arXiv:1512.01606 (2015).

${ }^{25}$ J. K. Gamble, M. A. Eriksson, S. N. Coppersmith, and M. Friesen, Phys. Rev. B 88, 035310 (2013).

${ }^{26}$ Y. Nakamura, Y. A. Pashkin, and J. Tsai, Nature 398, 786 (1999).

${ }^{27}$ M. Stopa, Phys. Rev. B: Condens. Matter 54, 13767 (1996).

${ }^{28}$ J. K. Gamble, N. T. Jacobson, E. Nielsen, A. D. Baczewski, J. E. Moussa, I. Montaño, and R. P. Muller, Phys. Rev. B 91, 235318 (2015).

${ }^{29}$ S. M. Goodnick, D. K. Ferry, C. W. Wilmsen, Z. Liliental, D. Fathy, and O. L. Krivanek, Phys. Rev. B 32, 8171 (1985). 